\title{
Congenital diverticulum of the right atrium situated on the floor of the coronary sinus
}

\author{
A PETIT, J C EICHER, P LOUIS \\ From the Department of Cardiology, Bocage University Hospital, Dijon, France
}

SUMMARY A diverticulum of the right atrium was found on the floor of the coronary sinus in a neonate. The diverticulum was distinguished from a pericardial cyst by contrast echocardiography, and its relations with coronary sinus and coronary venous return were identified by coronary angiography. Because the diverticulum compressed the left ventricular inferior wall and prevented its growth, it was excised. Thebesian veins were discovered at operation and prevented complete correction of the defect.

Congenital diverticula of the right atrium are rare anomalies, whereas idiopathic dilatation of the right atrium is more commonly reported. The coronary sinus may sometimes be slightly enlarged if it is receiving a left superior vena cava or total anomalous pulmonary venous return. Sizeable dilatation of the coronary sinus is uncommon and has been reported only once before. ${ }^{1}$ In this case, the right superior vena cava was absent, the persistent left vena cava drained the whole superior venous flow; the patient was symptom free.

We report on a diverticulum that we found at an unusual site in a neonate. To our knowledge this is the second case of this anomaly to be described and the first one in which an operation was performed.

\section{Case report}

A $3 \mathrm{~kg}$ baby boy was born by caesarean section after a 37 th week twin gestation. He had talipes, and a precordial murmur was heard when he was 10 days old. His brother was normal. The patient was referred to the pediatric cardiology unit. Physical examination showed a symptom free baby without cyanosis, with a grade $4 / 6$ pansystolic murmur and a single normal second heart sound. Chest radiography showed slight enlargement of the heart with a bulge from the right atrium and a slight prominence of the pulmonary vasculature. The electrocardiogram showed left axis deviation of $45^{\circ}$, an incomplete right bundle branch block, and a QS feature in leads II, III, and aVF. Echocardiography and pulsed Doppler

Requests for reprints to Dr A Petit, Centre de Cardiologie, Hôpital du Bocage, Dijon 21000, France. were performed. We found a small perimembranous ventricular septal defect and an ostium secundum. But the most striking discovery was the presence of a large cavity behind the posterior wall of the left ventricle. In the parasternal long axis view, this pouch seemed to reduce the tranverse diastolic diameter of the left ventricle, which measured only $18 \mathrm{~mm}$ (fig 1a). In the subcostal view, this pouch could be seen to extend down to the inferior aspect of the heart (fig lb). Because Doppler echocardiography did not detect any flow within this cavity, it was impossible to say whether the pouch was connected to any of the heart cavities or whether it was a pericardial cyst. Contrast echocardiography was performed after injection of dextrose into a left antecubital vein. Contrast echoes entered the right atrium immediately and then were quickly seen in the pouch, but never in the coronary sinus. This showed that the left superior vena cava had not persisted. A posterior diverticulum of the right atrium was diagnosed.

During the next few months the boy grew normally and remained symptom free. The ventricular septal defect closed spontaneously when he was six months old. But repeat echocardiograms showed that the size of the pouch remained the same and seemed to prevent growth of the left ventricle. When he was eight months old the ventricle was the same size as in the neonatal period and left ventricular filling was shown to be abnormal by pulsed Doppler examination. The peak velocity of atrial systole equalled that in early diastole. Cardiac catheterisation was performed when he was nine months old. Pressures inside the right cavities were normal. A left to right shunt was found only at the atrial level $(\mathrm{QP} / \mathrm{QS}=2)$. In the 

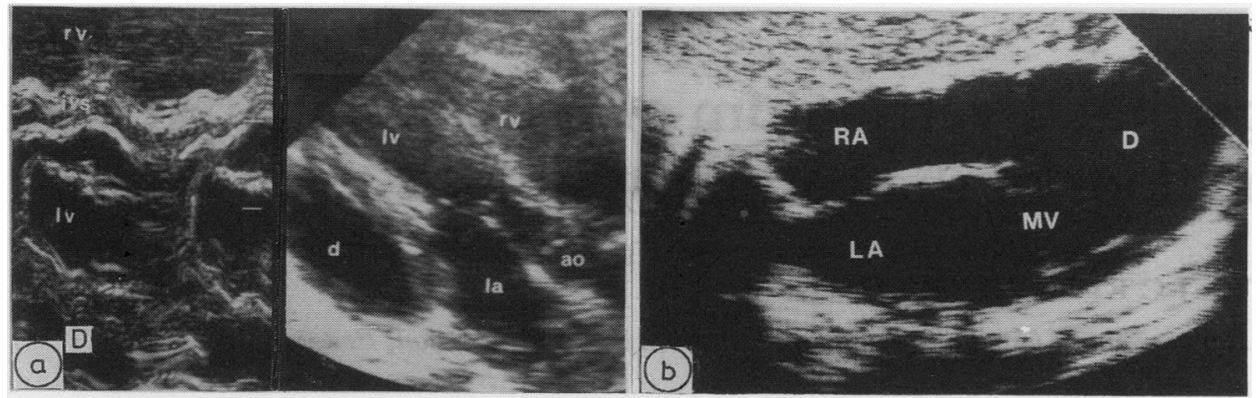

Fig 1 (a) $M$ mode and cross sectional echocardiograms in the parasternal long axis view. The diverticulum lies behind the left ventricle. (b) Cross sectional subcostal view through the inferior part of the heart into which the diverticulum extends. $D, d$, diverticulum; rv, right ventricle; lv, left ventricle; la, left atrium; ao, aorta; $M V$, mitral valve; $R A$, right atrium.

right atrium the catheter could be passed from the top to the floor through an atrial septal defect, through the tricuspid valve, and into a cavity in the left side of the chest in the anteroposterior view. The coronary sinus and left superior cava were not entered. The pressure and oxygen content of blood were the same in this pouch as in the middle of the right atrium. Left ventricular end diastolic pressure was normal $(10 \mathrm{~mm} \mathrm{Hg})$. Angiography showed that the diverticulum lay below and behind the heart and communicated with the right atrium through a large opening (fig 2a). Left ventricular injection confirmed the restriction of the posterior wall, the position of which was abnormally high (fig $2 b$ ). There was no evidence of a connection between the right ventricle and the pouch. An intracardiac electrocardiogram was performed to locate the electrophysiological pathways. There was no anomaly either in the right atrium or in the right ventricle. A QS feature was recorded inside the diverticulum as well as at the inferior wall of the heart. This feature was identical with the same QS feature in surface leads II, III, and aVF.

The anatomy of the coronary sinus was studied by selective coronary angiography. The coronary veins drained normally into the coronary sinus, which was not enlarged and overhung the opening of the diverticulum inside the right atrium.

Surgical correction was undertaken at the age of 11 months. The diverticulum was found to extend over

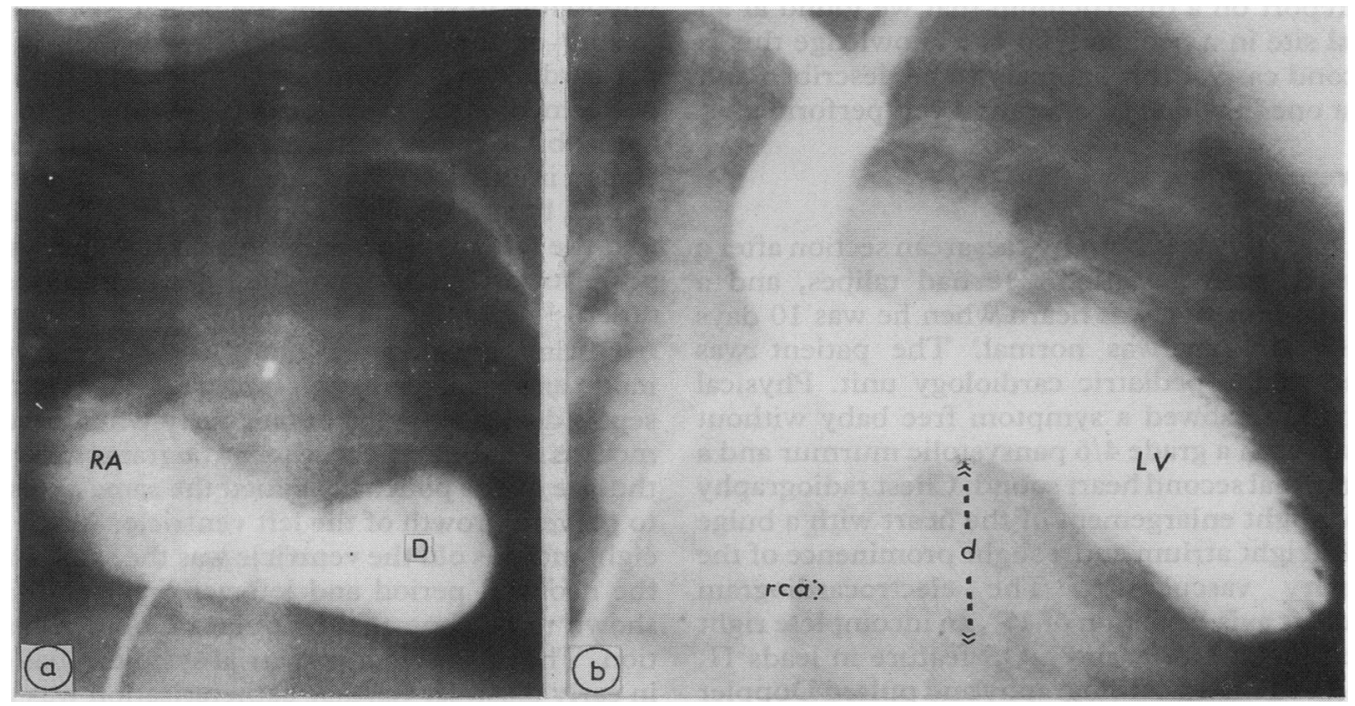

Fig 2 Cineangiograms showing (a) anteroposterior view after injection of contrast into the diverticulum and (b) $30^{\circ}$ right anterior oblique view of the left ventricle, which is raised up by the diverticulum which lies between the posterior wall of the ventricle and the right coronary artery ( $r c a)$. 
all the diaphragmatic area of the heart. There was a $1 \mathrm{~cm}$ diameter opening between the tricuspid valve and the ostium of the inferior vena cava. Some thebesian veins drained into the inlet of the pouch and therefore prevented complete closure of the defect at operation. The distal four fifths of the diverticulum were closed by resection of the redundant tissue and with a padding suture. The atrial septal defect was then closed. At the end of the operation a tiny cavity remained at the junction between right atrium and left ventricle inferior wall to receive the coronary sinus and thebesian veins. Histological examination of the diverticulum wall showed that it was made of normal atrial tissue.

The postoperative course was uneventful. Three months later physical examination was normal. The radiographic enlargement of the heart had decreased. The electrocardiogram showed that the $Q S$ feature in the frontal leads had disappeared and the electrical axis view had become perpendicular to the frontal plane. Echocardiography in parasternal long axis view showed the absence of the cavity and that the left posterior wall adjoined the pericardium. The subcostal view showed a tiny pouch behind the tricuspid valve and the interventricular septum. Examination by pulsed Doppler showed that left ventricular filling was normal and that the diameter of the ventricle in diastole had increased to $30 \mathrm{~mm}$.

\section{Discussion}

This diverticulum developed at the junction between the coronary sinus and the right atrium. We have found only one similar case in published reports. ${ }^{2}$ It was of a neonate with a hypoplastic left ventricle who died at the age of four days. The echocardiogram and necropsy showed a large diverticulum behind the left side of the heart with an opening on the floor of the coronary sinus into which a left superior vena cava drained.

The explanation for the diverticulum may lie in embryological development. The right Cuvier's canal becomes the right superior and inferior vena cavae while the left canal usually regresses after the fourth week of pregnancy to become the coronary sinus or sometimes a persistent left superior vena cava. ${ }^{3}$ In Di Segni's case, as in ours, incomplete regression of the left Cuvier's canal may explain the presence of a retrocardiac pouch. Furthermore, the hypoplastic left ventricle in Di Segni's case and the failure of our patient to thrive suggest that such a diverticulum causes compression and leads to under- development of the left ventricle. This is why we recommended early excision of the diverticulum.

Another possible site for a diverticulum of the right atrium is anterior, on the appendage. There is one report of such a case, in which an enormous diverticulum covered the anterior side of the heart, and caused severe episodes of arrhythmia when the patient was 16 . The cavity contained a thrombus and the patient recovered after its removal. ${ }^{4}$

We would expect the prognosis when a diverticulum is present to resemble that of idiopathic dilatation of the right atrium, which is a more common condition. The parietal wall of a diverticulum contains normal amounts of atrial muscle fibres, as in our case, or slightly reduced amounts, ${ }^{4}$ but in idiopathic dilatation of the right atrium the parietal tissue is almost devoid of muscle fibres. ${ }^{5}$ Although it was initially regarded as benign, idiopathic dilatation has been found to be associated with congestive heart failure, ${ }^{6}$ arrhythmias, ${ }^{7}$ and sudden death. ${ }^{8}$

\section{References}

1 Mossard JM, Dimitrov N, Kim K, Voegtlin R, Bloch P. Retour veineux cave anormal avec ectasie de l'oreillette droite et du sinus coronaire. Ann Radiol 1979;22,7:605-7.

2 Di Segni E, Siegal A, Katzenstein M. Congenital diverticulum of the heart arising from the coronary sinus. Br Heart J 1986;56:380-4.

3 Ben Pansky $\mathrm{Ph}$ B. In: Horn FH, ed. Embryologie humaine. New York: Ellipses Marketing Edition, 1986:308-9.

4 Morrow AG, Behrendt DM. Congenital aneurysm (diverticulum) of the right atrium. Clinical manifestations and results of operative treatment. Circulation 1968;38:124-8.

5 Garcia MJ, Allen RJ. Idiopathic dilatation of the right atrium. Postoperative follow up in a child. $J$ Pediatr Surg 1983;18,2:196-8.

6 Sheldon WC, Johnson CD, Favaloro RG. Idiopathic enlargement of the right atrium. Report of four cases. Am J Cardiol 1969;23:278-84.

7 Slama R, Coumel Ph, Perrault MA. Dilatation idiopathique de l'oreillette droite avec troubles du rythme et paralysie auriculaire apparente. Arch Mal Coeur 1973;66:35-47.

8 Tenckoff L, Stamm SJ, Beckwith JB. Sudden death in idiopathic congenital right atrial enlargement. Post mortem findings and review of cases. Circulation 1969;40:227-35. 